\title{
Salud oral en pacientes con diabetes tipo 2: caries dental, enfermedad pe- riodontal y pérdida dentaria
}

Oral health in patients with diabetes type 2: dental caries, periodontal disease and tooth loss

\begin{abstract}
Resumen
El propósito de este estudio fue determinar la relación entre la salud bucodental y el grado de control metabólico entre sujetos con diabetes mellitus tipo 2 no insulino dependiente. Trescientos sujetos fueron divididos en dos grupos de 150: Grupo I, con control metabólico (hemoglobina glucosilada [HbA1c] < 8.0\%) y Grupo II, sin control metabólico (HbA1c $\geq 8.0 \%$. La examinación clínica incluyó frecuencia de prácticas de higiene oral, estado periodontal usando el Índice Comunitario Periodontal de Necesidades de Tratamiento (ICPNT), Índice de Caries de Necesidades de Tratamiento (ICNT) y el número de dientes perdidos. Una correlación positiva y estadísticamente significativa fue observada entre los dos indicadores de salud bucodental (CITN and CPITN) y la duración de la enfermedad y el grado de control metabólico. El estudio demostró que los sujetos con diabetes tendrían que mejorar sus prácticas de higiene oral, abandonar el hábito de fumar, y controlar los niveles de glucosa sanguínea.
\end{abstract}

\begin{abstract}
The aim of this study was to determine the relationship between bucco-dental health and the degree of metabolic control among type II non-insulin dependent diabetic mellitus subjects. Three hundred subjects were divided into two groups of 150: Group I, diabetic with metabolic control (glycosylated haemoglobin $[\mathrm{HbA1c}]<8.0 \%$ ) and Group II, diabetic without metabolic control (HbA1c $\geq 8.0 \%$ ). Clinical examination included frequency of oral hygiene practices, periodontal status by using the Community Periodontal Index of Treatment Needs (CPITN), Caries Index Treatments Needs (CITN), and the number of missing teeth. A statistically significant positive correlation was observed between two bucco-dental health indicators (CITN and CPITN) and both the duration of the disease and the degree of metabolic control. The study indicated that diabetic subjects should improve their oral hygiene practices, discontinue cigarette smoking and control blood glucose levels.
\end{abstract}

\section{Introducción}

La diabetes es una afección cuya irreversibilidad y permanencia en el organismo de una persona, la definen como una enfermedad crónico-degenerativa. Existen diferentes tipos: a) tipo 1, aproximadamente, $5 \%$ de los casos; b) tipo 2, 90-95\% de los casos; c) asociada con otras condiciones específicas o síndromes; d) daño en la tolerancia a la glucosa y en la glucosa en ayunas; e) gestacional. ${ }^{1}$

De acuerdo a las proyecciones de la OMS, tendrá un incremento de la prevalencia en adultos de un $42 \%$ en los países desarrollados y de un $170 \%$ en países en vías de desarrollo. Para el año 2025 el 75\% de la población de diabéticos en el mundo residirá en algún país en desarrollo. ${ }^{2}$

La diabetes mellitus tipo 2 (DT2) afecta a personas mayores de 40 años, frecuentemente obesas o con sobrepeso. Esta variedad de enfermedad metabólica se caracteriza por el déficit parcial de insulina que se manifiesta por alteraciones en el metabolismo de la glucosa. ${ }^{3}$

Se ha convertido en un problema de salud pública a nivel mundial, pues produce un alto impacto económico y social, ocasionando la pérdida de años de vida productiva. ${ }^{4}$

Las patologías más frecuentes, a nivel

\section{Rolando Pablo Juárez ${ }^{1,2}$, Julio Ricardo Chahín', Maytena Margarita Vizcaya², Emma Isabel Arduña²}

Facultad de Odontología. Universidad Nacional del Nordeste, Argentina

Hospital Central de Odontología. Ministerio de Salud Pública de la Provincia del Chaco, Argentina

Correspondencia:

Dr. Rolando Juárez. Profesor Adjunto de la Cátedra de Fisiología Humana Facultad de Odontología. Universidad Nacional del Nordeste. Argentina Av. Rivadavia 862. (3500) Resistencia, Chaco. Argentina. TEL: 03722 - 420996. e-mail: rolandojuarez@gigared.com

Palabras clave: Diabetes tipo 2, nivel de glucosa sanguínea, higiene oral, caries, ICNT, enfermedad periodontal, ICPNT, hábito de fumar.

Key words: Diabetes type 2, blood glucose level, oral hygiene, decay, CITN, periodontal disease, CPITN, smoking. estomatológico, son la enfermedad periodontal, caries, candidiasis, mucormicosis, queilitis comisural, xerostomía y síndrome de ardor bucal. ${ }^{5}$

El objetivo de este trabajo es determinar la relación de la morbilidad bucal con el estado de control metabólico en pacientes diabéticos tipo 2 de la consulta de periodoncia del Hospital Central de Odontología de la ciudad de Resistencia.

\section{Materiales y Métodos}

Para dar cumplimiento al objetivo propuesto se procedió a seleccionar el diseño muestral, que consistió en pa- 
cientes con diagnóstico de diabetes que acudieron a una consulta programada de periodoncia del Hospital Central de Odontología, en el período de dos años (junio 2004 - julio 2006), clasificados previamente como pacientes con DT2.

Previo consentimiento informado, se recogieron datos en protocolo elaborado a tal fin que incluyeron datos de filiación, datos sociodemográficos, grado de control metabólico empleando las determinaciones de hemoglobina glicosilada, duración de la diabetes, hábitos higiene oral y de fumar.

Finalmente la muestra quedó conformada por 300 pacientes, de ellos 150 con control metabólico (Grupo I, hemoglobina glucosilada [HbA1c] $<$ $8.0 \%$, media $6.7 \%$ ) y 150 sin control metabólico (Grupo II, HbA1c $\geq 8.0 \%$, media $13,7 \%$ ).

El examen clínico de la cavidad bucal se realizó con un espejo plano, una sonda periodontal (con los requerimientos estipulados por la OMS) y un explorador Nro. 5, siguiendo criterios establecidos para la aplicación de los índices: 1) Necesidad de tratamiento de caries dental (INTCD, Bordoni y col., 1994), 2) Índice comunitario de necesidad de tratamiento periodontal (ICNTP, Ainamo y col., 1982), citados en el Programa de educación continua odontológica no convencional (PRECONC). ${ }^{6}$

La información recabada a partir de la exploración bucal realizada fue registrada en una hoja de valoración individual. Posteriormente, esta información fue guardada en una base de datos.

El análisis estadístico se realizó con el programa para análisis estadístico SAS (Statistical Analysis System), en base a distribución de frecuencias, media y desviación estándar, test de diferencia de proporciones, comparación de medias (t-test) y análisis de correlación con un nivel de significancia de $p<0.05$. Todas las pruebas se realizaron a un nivel de confianza del $95 \%$.

\section{Resultados}

Los 300 pacientes tenían un intervalo de edad entre 40 y 65 años, con promedio de: Grupo I, pacientes controlados, edad media $=56.1 \pm 3.1$ años; Grupo II, descontrol metabólico, edad media $=55.4 \pm 3.2$ años.

En la distribución por sexos, de los 150 pacientes controlados 96 eran mujeres, es decir un $64 \%$ y 54 varones, lo que representa un $36 \%$.
Paralelamente de los no controlados 51 eran mujeres (34\%) y 99 hombres $(66 \%)$.

\begin{tabular}{|l|c|c|c|}
\hline \multicolumn{3}{|c|}{ Tabla I. Características (\%) de pacientes con CM y sin CM } \\
\hline & $\mathrm{C}$ & $\begin{array}{c}\mathrm{NC} \\
\mathrm{n}=150\end{array}$ & Valor de $\mathrm{p}$ \\
\hline Educación & 31 & & $<0.0001$ \\
Básica & 46 & 64 & $<0.001$ \\
Media & 23 & 13 & $<0.05$ \\
Terciaria y Superior & 65 & 34 & 0.0001 \\
Estado Civil & 4 & 24 & $\mathrm{~N}$ \\
Casado/a & 25 & 37 & $\mathrm{NS}$ \\
Separado/a & 6 & 5 & $<0,01$ \\
Unión Estable & 46 & 27 & $\mathrm{NS}$ \\
Viudo/a & 30 & 41 & $\mathrm{NS}$ \\
Estado Laboral & 24 & 31 & $<0.01$ \\
Ocupados & 39 & 21 & \\
Desocupados & & & \\
Inactivos & & & \\
Obra social con & & & \\
cobertura odontológica & &
\end{tabular}

Control metabólico: C, controlado; NC, no controlado; NS, no significativas.

\begin{tabular}{|l|c|c|c|}
\hline \multicolumn{2}{|c|}{ Tabla II. Comportamiento (\%) de los pacientes diabéticos, con respecto a su salud bucal } \\
\hline & $\mathrm{N}=150$ & $\begin{array}{c}\mathrm{NC} \\
\mathrm{n}=150\end{array}$ & Valor de $\mathrm{p}$ \\
\hline Cepillado & 3 & & \\
No & 32 & 13 & $<0.01$ \\
1 vez por día & 47 & 53 & $<0.01$ \\
2 veces por día & 17 & 30 & $<.01$ \\
Más de 2 veces por día & & 4 & 0.001 \\
Hilo Dental & 28 & 53 & $<0.001$ \\
No & 21 & 37 & $<0.01$ \\
1 vez por día & 35 & 6 & $<0.01$ \\
2 veces por día & 16 & 5 & 0.01 \\
Más de 2 veces por día & & & $<0.01$ \\
Hábito de Fumar & 26 & 41 & NS \\
Fumador & 39 & 21 & \\
Fumador Pasado & 34 & 38 & \\
No Fumador & & & \\
\hline
\end{tabular}

Control metabólico: $C$, controlado; NC, no controlado; NS, no significativas.

\begin{tabular}{|c|c|c|c|}
\hline \multicolumn{4}{|c|}{ Tabla III. Índice de necesidad de tratamiento de caries dental (\%) } \\
\hline & $\begin{array}{c}C \\
N=150\end{array}$ & $\begin{array}{c}\text { NC } \\
\mathrm{n}=150\end{array}$ & Valor de $\mathrm{p}$ \\
\hline $\begin{array}{l}\text { Valor } 11 \text { (ausencia de dientes } \\
\text { en } 1 \text { cuadrante de la boca). } \\
\text { PPA, TP, E, RP en } 1 \text { cuadrante } \\
\text { Valor } 12 \text { (ausencia de } \\
\text { dientes en } 2 \text { cuadrantes de } \\
\text { la boca). PPA, TP, E, RP en } 2 \\
\text { cuadrantes } \\
\text { Valor } 13 \text { (ausencia de } \\
\text { dientes en } 3 \text { cuadrantes de } \\
\text { la boca). PPA, TP, E, RP en } 3 \\
\text { cuadrantes } \\
\text { Valor } 14 \text { (ausencia de } \\
\text { dientes en } 4 \text { cuadrantes de } \\
\text { la boca). PPA, TP, E, RP en } 4 \\
\text { cuadrantes }\end{array}$ & 19 & 17 & $<0.01$ \\
\hline
\end{tabular}

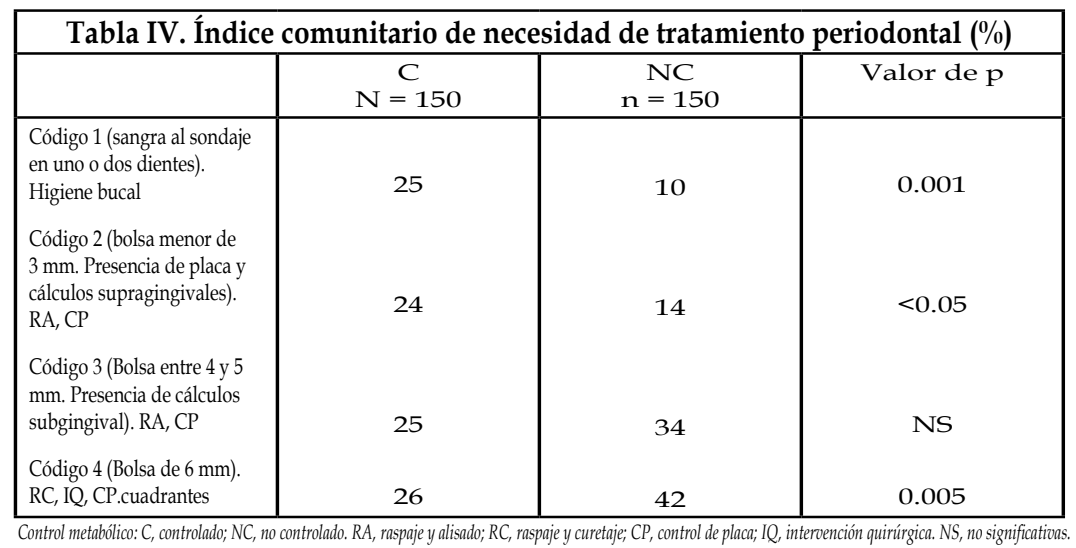


los pacientes controlados un nivel educativo superior, un predominio de casados, mayor tasa de ocupación laboral y apropiada cobertura de obra social.

Respecto a las variables de antecedente personal patológico, encontramos que la duración de la diabetes en nuestros pacientes fue de: 1) pacientes controlados = $7,1 \pm 2,6$ años; 2 ) pacientes no controlados $=11,5 \pm 9,2$ años $(t=5,637 ; p<0,0001)$.

En la Tabla II, se exhibe el comportamiento de los pacientes con respecto a su salud oral. Ausencia y/o baja frecuencia de cepillado y uso de hilo dental se observó en los pacientes no controlados. Al momento de la investigación, los pacientes fumadores predominaron dentro de los no controlados y los que dejaron de fumar dentro de los controlados. En los no fumadores no hubo diferencias significativas entre ambos grupos.

En la Tabla III, se observa como se distribuyeron los pacientes según los criterios del INTCD, repartiéndose la condición de salud entre los valores del índice 11 y 14 . No se encontraron diferencias significativas entre ambos grupos de pacientes, salvo el valor 11 donde la condición de salud fue deficiente en los pacientes controlados.

En la Tabla IV, se presenta el número de pacientes diabéticos controlados y no controlados, afectados por enfermedad periodontal. Se puede apreciar que el grupo de pacientes diabéticos no controlados presentó un mayor ICNTP correspondiente al código 4, mientras que en los códigos 1 y 2, predominaron los diabéticos controlados. En el código 3 , no se observaron diferencias significativas entre ambos grupos.

Al estudiar la asociación entre el INTCD y ICNTP, se encontró una correlación significativa entre ambos grupos, en los pacientes controlados $(\mathrm{r}=0,987 ; \mathrm{p}=0,01)$ y no controlados $(\mathrm{r}$ $=0,989 ; \mathrm{p}=0,01$ ).

En los pacientes no controlados se observó una asociación significativa entre los valores de $\mathrm{HbA} 1 \mathrm{c}$ con: el INTCD $(\mathrm{r}=0,962 ; \mathrm{p}=0,04)$ y el ICNTP $(\mathrm{r}=$ $0,974 ; p=0,02)$; así como los códigos del ICNTP con: el hábito de fumar ( $\mathrm{r}$ $=0,957 ; p=0,03)$ y la evolución de la enfermedad $(r=0,951 ; p=0,03)$.

El registro de la pérdida dentaria mostró 2.921 piezas dentarias ausentes, siendo la media por persona en pacientes controlados de $3,5 \pm 1,1$ y no controlados de $9,7 \pm 2.1(\mathrm{t}=32,031 ; \mathrm{p}<0,0001)$.

Con indicación de exodoncia había 827 piezas dentarias, 629 por enfermedad periodontal y 198 por caries. El $85 \%$ de los dientes con enfermedad periodontal y el $49 \%$ de los dientes con caries, pertenecían a pacientes no controlados.

\section{Discusión}

La división en dos grupos de los pacientes estudiados, a través del control metabólico, nos permitió identificar el impacto clínico de la hiperglucemia sobre la salud oral. Utilizamos como grupo control, a los pacientes controlados, basándonos en la evidencia científica. Investigaciones han determinado que la salud oral y la respuesta tisular al tratamiento odontológico en el paciente diabético controlado son similares al sano. ${ }^{7}$

La utilización de la hemoglobina glucosilada [HbA1c] en este estudio nos aseguró un riguroso control glucémico, pues representa hasta el momento la mejor prueba de laboratorio que determina si la diabetes se tiene bajo control, mostrando un estimado del promedio del nivel de glucemia en las 6 a 10 semanas previas; adjudicándose valores aceptables ("moderados") en un porcentaje $<8 \%$, según los criterios del Consenso Europeo.

En los dos grupos de diagnóstico de este estudio, la frecuencia se incrementó al aumentar la edad, un hallazgo similar al informado en un estudio realizado entre 1976 y 1991 por la OMS, el cual es más evidente en las naciones consideradas de moderado a alto riesgo para diabetes; el pico máximo se presentó en la sexta década de la vida, sin embargo, en algunos países de América Latina, es más alto en los grupos de más edad.

La prevalencia de DM generalmente no muestra diferencias estadísticas significativas entre hombres y mujeres. Se han buscado posibles explicaciones a este comportamiento de la enfermedad y la evidencia sugiere que no se relaciona con un sustento biológico intrínseco, dado que algunos países africanos la prevalencia es mayor en hombres, por lo que probablemente se asocie a determinantes de índole ambiental, tales como la obesidad, el sedentarismo y la dieta, entre otros. ${ }^{9}$

En nuestro estudio se encontró un predominio de mujeres dentro de los pacientes controlados y de hombres en los no controlados. Este hallazgo quizá este relacionado al mayor nivel educativo encontrado en las mujeres y a pautas culturales locales.

La realidad latinoamericana de bajas condiciones socioculturales y edu- cacionales, obliga a reconocer serias limitaciones para el control de la glucosa. ${ }^{10}$

Un nivel educacional bajo, puede influir en el grado de conocimiento de los factores de riesgo de la diabetes para su prevención, así como en el grado de interpretación, cumplimiento de las orientaciones médicas y prácticas de higiene. ${ }^{11}$ El menor nivel educativo apreciado dentro de los pacientes no controlados en este estudio, es un claro ejemplo de lo mencionado precedentemente.

La diabetes afecta los estilos de vida y aumenta la desocupación, debido a las complicaciones, minusvalías y las actividades necesarias para controlar la glucemia, de allí la necesidad de conocer el estado socioeconómico cuando se estudia el impacto de la patofisiología de esta enfermedad crónica sobre la salud oral. ${ }^{12}$ En nuestro estudio se observó una mayor estabilidad familiar y laboral, entre los pacientes controlados.

En la población general, la falta de obra social, particularmente cobertura odontológica, está asociado a un menor uso de los servicios dentales y a una salud oral deficiente. Como la obra social es un beneficio de empleo, las personas desocupadas posiblemente tendrán menor cobertura odontológica. ${ }^{13}$ Estas afirmaciones coinciden con nuestros hallazgos sobre la mayor cobertura odontológica de los pacientes controlados, quienes a su vez, presentaron una mejor condición de salud bucal.

La importancia del registro de la duración de la diabetes radica en que, junto al mal control glicémico están asociados a los diversos factores predisponentes de infección. ${ }^{14}$

La enfermedad periodontal en los individuos diabéticos se presentaría de forma similar a las complicaciones crónicas de la diabetes; es decir, a mayor duración de la diabetes mayor frecuencia de periodontitis. Por otra parte, la presencia de complicaciones avanzadas se correlaciona con una mayor severidad de la periodontitis. ${ }^{15}$ En nuestro estudio, observamos una asociación significativa entre los valores del ICNTP y la evolución de la diabetes, lo que estaría de acuerdo con lo publicado anteriormente.

Nuestros hallazgos de mayor frecuencia de cepillado y uso de hilo dental en los pacientes controlados, coinciden con publicaciones en donde se afirma que la higiene oral correcta tiene un efecto positivo en el control metabólico de los diabéticos T2. Pero, bajo las mismas condiciones de higiene oral, la diabetes afecta el metabolismo, causa disminu- 
ción de las defensas inmunitarias y perturba los mecanismos de reparación tisular; siendo necesario entonces incrementar las medidas de higiene oral. ${ }^{16}$

La frecuencia de los signos bucales de la enfermedad en los pacientes diabéticos en el presente estudio se semeja de modo importante con las reportadas por otros autores. La asociación de la diabetes con la pérdida dentaria es generalmente aceptada, pero existe una controversia en cuanto al aumento de la presencia de caries en pacientes diabéticos. ${ }^{17}$ En este estudio, en el grupo de los pacientes no controlados se observó un incremento progresivo del empeoramiento de la condición de salud, según se observa en la Tabla III, INTCD.

Nuestros hallazgos coinciden con la mayoría de los trabajos publicados en la literatura internacional, que consideran a la diabetes como un factor de riesgo para la enfermedad periodontal, siendo la severidad tanto mayor, cuando peor es el control metabólico. ${ }^{18} \mathrm{Del}$ análisis de la Tabla IV (ICNTP), se desprende que el porcentaje de pacientes controlados, mostró una distribución casi equilibrada en los diferentes códigos del índice, mientras que los no controlados predominaron en los códigos 3 y 4 , indicativos de mayor severidad periodontal.

Además, existe cierta evidencia de que la enfermedad periodontal severa, puede deteriorar el control de la glucemia, mientras que una adecuada terapia periodontal mejora el metabolismo de la glucosa. ${ }^{19}$ Quizás, el descubrimiento más importante de nuestros resultados, fue que los pacientes con DT2 y enfermedad periodontal severa, tenían un pobre control de su glucemia, como indicó la alta concentración de HbA1c.

Es conocida la relación entre enfermedad periodontal y el tabaco. Estudios han demostrado que fumar incrementa el riesgo de padecer enfermedad periodontal en pacientes diabéticos. ${ }^{12}$ La mayoría de los pacientes no controlados, de este estudio, eran fumadores y a su vez estos se encontraban en mayor número, dentro de los códigos 3 y 4 del ICNTP.

La asociación positiva encontrada entre el componente cariado del índice y el CPITN, posiblemente, se deba a la presencia de placa dentobacteriana, ya que ambos padecimientos tienen relación con este depósito.

En este estudio, el mayor número de piezas dentarias pérdidas y de dientes con indicación de exodoncia por enfermedad periodontal, se observa en pacientes diabéticos con deficiente control metabólico. La pérdida dentaria tiene un efecto adverso sobre la salud general, debido a sus consecuencias en la elección e ingesta de alimentos sobre la capacidad del paciente para mantener una dieta saludable y controlar apropiadamente su glucemia. ${ }^{20}$

Al término del estudio se llegó a las siguientes conclusiones:

El estado de higiene y salud periodontal de la población diabética tipo 2 incluida en nuestro estudio, mostró diferencias de acuerdo al control metabólico del paciente.

Un control metabólico inadecuado, incrementó la severidad de la enfermedad periodontal y la necesidad de tratamientos más complejos. Los pacientes que regularmente controlaban su enfermedad, tenían mejores hábitos de higiene bucal y una adicción al tabaco menor que los pacientes no controlados, disminuyendo el riesgo de presentar enfermedad periodontal.

El equipo de salud tendría que enfatizar los beneficios de realizar un control odontológico periódico, practicar una efectiva higiene oral y suspender el hábito de fumar, especialmente en aquellos pacientes con un control metabólico inadecuado.

\section{Referencias bibliográficas}

1. American Diabetes Association. Report of the Expert Committee on the Diagnosis and Classification of Diabetes Mellitus. Diabetes Care 1997;20: 1183-1197.

2. King $H$, Aubert RE and Herman WH. Global burden of diabetes, 1995-2025: prevalence, numerical estimates, projections. Diabetes Care 1998; 21:1414-1431.

3. Silvestre Donat J, Sanchis Bielsa JM, Cutando Soriano D. El paciente diabético en la clínica dental. Rev Act Odontoestomatol Esp 1995;7: 56-61.

4. Chatuverdi N, Jarret J, Shipley MJ, Fuller JH. Socioeconmic gradient in morbidity and mortality in people with dabetes: cohort study findings from the Whitehall study and the WHO multinational stuy of vascular disease in diabetes. British Medical Journal 1998; 316:100-105.

5. Gay Zárate O. Actualidades en el manejo dental del paciente diabético. ADM 1999; LVI: 18-26.

6. Bordoni N. Planificación. Diagnóstico de necesid ad de tratamiento para afecciones bucales prevalentes. En: Programa de Educación Continua Odontológica No Convencional. Curso 4 , gestión del componente salud bucal de la atención de salud. Módulo 1, planificación-programación del componente salud bucal. Publicación de la Organización Panamericana de la Salud, 1998.
7. Ogunbodede EO, Fatusi OA, Akintomide A, Kolawole K, Ajayi A. Oral Health Status in a Population of Nigerian Diabetics. J Contemp Dent Pract 2005;6:075-084.

8. Alberti KG, Gries FA, Jervell J, Kraus $\mathrm{HM}$. A desktop guide for management of non-insulindependent diabetes mellitus (NIDDM): an update. Diab Med 1994;11:899-909.

9. Roselló-Araya M. Prevalencia de diabetes tipo 2, intolerancia a la glucosa y diabetes provisional en El Guarco, Cartago. Rev Costarric Cienc Med 2003;24:15-24.

10.Jiménez M. Diabetes Mellitus: actualización. Acta Médica Cost 2000;42:53-65.

11. Villena J, Romero S. Características socioeconómicas y culturales de los pacientes diabéticos no insulinodependientes del Hospital Nacional Cayetano Heredia. Diagnóstico 1991;28:93-7.

12. Moore PA, Weyant RJ, Mongelluzzo MB, Myers DE, Rossie K, Guggenheimer $\mathrm{J}$ et al. Type 1 Diabetes Mellitus and Oral Health: assessment of periodontal disease. J Periodontol 1999; 70:409-417.

13. Damiano PC, Shugars DA, Johnson JD. Expanding health insurance coverage and the implications for dentistry. J Public Health Dent 1992;52:52-8.

14. Nathan DM. Long-term complications of diabetes mellitus. N Engl J Med 1993;328: 1676-83

15. Miller LS, Manwell MA, Newbold D, Reding ME, Rasheed A, Blodgett J et al. The relationship between reduction in periodontal inflammation and diabetes control: a report of 9 cases. J Periodontol 1992;63:843-8.

16. Almas K, Al-Lazzam S, Al-Quadairi A. The Effect of Oral Hygiene Instructions On Diabetic Type 2 Male Patients With Periodontal Diseases. J Contemp Dent Pract 2003,3:040-051.

17. Cruz Peternell LR, Noble Solís FE, García Alcalá H. Manifestaciones bucales de diabetes mellitus: experiencia en el Hospital General de Puebla. Practica Odontológica 1995;16:5-7.

18. Lalla E, Lamster IB, Schmidt AM. Enhanced interaction of advanced glycation end products with their cellular receptor RAGE: implications for the pathogenesis of accelerated periodontal disease in diabetes. Ann Periodontol 1998;3:13-9.

19. Almas K, Al-Quahtani M, Al-Yami M, Khan N. The Relationship Between Periodontal Disease And Blood Glucose Level Among Type II Diabetic Patients. J Contemp Dent Pract 2001;2:18-25.

20. Taylor GW, Manz MC, Borgnakke WS.

Recibido : 23-04-2007

Aceptado para publicación: 25-05-2007 